\title{
RECHERCHES SUR LA PASTEURISATION BASSE DU LAIT. UNE NOUVELLE RÉACTION POUR CONTROLER LA TEMPÉRATURE DU CHAUFFAGE
}

\author{
par le Professeur ORLA-JENSEN \\ $D^{r}$ en philosophie et $D^{r}$ es-Sciences,
}

avec la collaboration de C. LE DOUS, Johanne JACOBSEN et N.C. OTTE.

(Suite et fin)

\section{TENEUR DU LAIT EN BACTÉRIES.}

Dans les essais de pasteurisation, les numérations directes des bactéries sous le microscope sont exclues, car on ne peut pas distinguer les cellules vivantes des mortes. On ne peut dans ces conditions qu'utiliser la numération sur plaques, et comme milieu alimentaire, nous avons employé la gélatine avec de la caséine peptcnicée et du glucose, décrit dans ma bactériologie laitière, et qui est un milieu spécialement favorable aux bactéries du lait. Les numérations furent constamment exécutées quand les plaques étaient restées une semaine à $20^{\circ} \mathrm{C}$. Pour l'ensemencement, on a également employé la méthcde décrite dans'ma bactériologie laitière, dans laquelle on ne mesure pas moins $\mathrm{d}^{\prime} 1 / 2 \mathrm{~cm}^{3}$ du lait ou des dilutions et jamais"un certain ncmbre de gouttes, qui peuvent avoir des dimensions très diverses d'après la nature de la pip€t1e, ou la façon dont on la tient.

Des essais préliminaires ont montré que lor que la durée du chauffage n'est pas plus longue que 30 minutes, il n'y a pas de diminution sensible de la teneur en bactéries avant $47^{\circ} 5 \mathrm{C}$. Dans le 1ableau XLV, on voit comment le nombre des germes diminue au fur et à mesure que la température et la durée du chauffage avgmentent.

Comme d'autres chercheurs qui se sont occupés antérieurement de l'influence de la pasteurication sur le nombre des geımes du lait, nous avons aussi calculé le pourcentage du nombre originel de germes résistant à la pasteurisation. Ceci est pourtant tout à fait sans valeur, car ce nombre ne dépend pas seulement de la nature des bactéries, mais surtout et beaucoup du nombre des germes originellement dans le lait, car, dans les diverses méthcdes de chauffage, nous arrivons presque toujours à la même teneur en bactéries, que le lait soit riche ou non en bactéries. Ainsi, si nous nous en tenons au lait ayant subi la pasteurication lente habituelle (chauffage à $63^{\circ} \mathrm{C}$. pendant 30 minutes), le $0 / 00$ des survivantes n'est, d'après le tableau XLV, que 0,1 pour le plus mauvais lait (1re colonne), tandis qu'il est 10 , soit 100 fois plus grand pcur le meilleur lait ( $3^{\mathrm{e}}$ colonne). Comme pratiquement toutes les bactéries qui peuvent se développer à basse température sont tuées par la pasteurication lente, il est tout à fait particulier que dans le lait conservélongtemps à basse température $\left(5-10^{\circ} \mathrm{C}\right.$.) la pasteurisation a gisse pour niveler le nombre des germes. Ceci ressort avec toute la clarté voulue du tableau LI. Si 
TABLEAU XLV

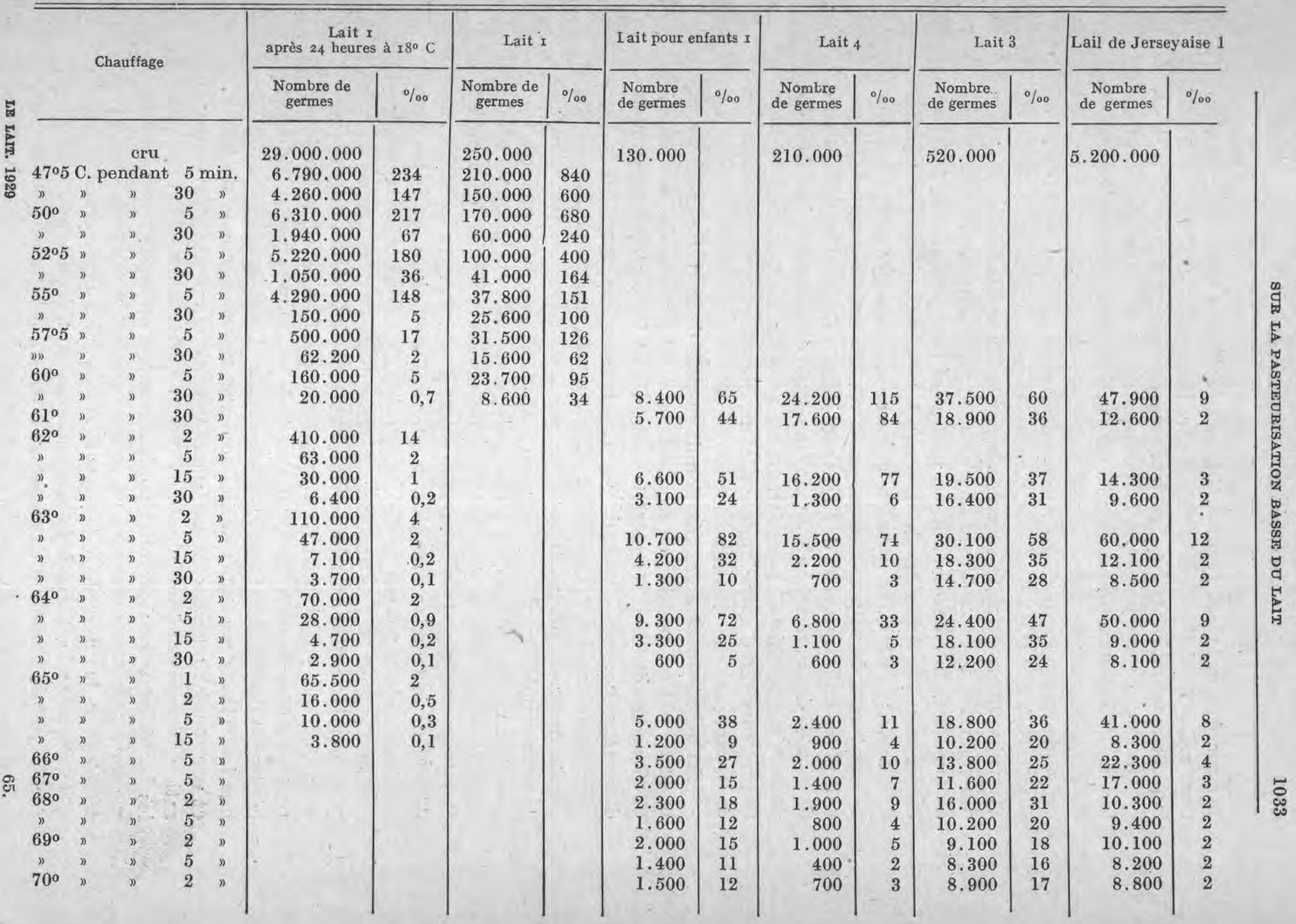


par conséquent le lait ayant subi la pasteurisation basse est seulement conservé au froid, il se tient au moins aussi longtemps que le lait ayant subi la pasteurisation haute. Nous reviendrons plus loinsur ce point important.

On sait parfaitement que la température et la durée du chauffage lors de la pasteurisation se remplacent partiellement et mutuellement, et il est intéressant de voir que par 5 minutes de chauffage à $68^{\circ} \mathrm{C}$. on arrive de très près au même nombre de germes que par 30 minutes de chauffage à $63^{\circ} \mathrm{C}$. Ceci se voit nettement dans le tableau XLVII, où, ayant justement ce point en vue, nous avons essayé le lait de 40 vaches différentes. Si par conséquent on n'attache pas d'importance à la faculté de montée de la crème du lait, on peut aussi bien pratiquer la pasteurisation basse par 5 minutes de chauffage à $68^{\circ}-69^{\circ} \mathrm{C}$. que par 30 minutes de chauffage à $63^{\circ} \mathrm{C}$. La première méthode est pratiquement plus rapide et plus commode, et peut, comme on l'a vu, être exécutée avec des appareils plus simples que celui de Stassano. Dans la destruction de la faculté de montée de la crème, on a un moyen símple de contrôler si le lait a été pour le moins chauffé aussi fortement, mais on n'a pas, comme nous l'avons aussi dit, d'autre limite supérieure que celle que donne la réaction de SToRor, et celle-ci est un peu trop élevée.

En mentionnant notre réaction de montée de la crème, nous avons montré qu'il est possible de fixer celle-ci à 30 minutes de chauffage à $63^{\circ} \mathrm{C}$, au lieu de $62^{\circ} \mathrm{C}$. Il ressort du tableau XLVI que généralement nous diminuons encore le nombre de germes à la moitié en pratiquant la pasteurisation lente à $63^{\circ} \mathrm{C}$. au lieu de $62^{\circ} \mathrm{C}$, mais que ceci, au point de vue absolu, ne signifie pas grand'chose. On pouvait certainement craindre que ce petit supplément de chauffage ait justement quelque signification en ce qui concerne les bactéries pathogènes, mais on a été jusqu'ici plutôt trop sévère que trop modéré dans les exigences sur ce point, parce qu'au lieu de se contenter d'une mise hors d'état de nuire de ces bactéries on a désiré leur destruction complète, ce qui est tout à fait inconciliable avec la nécessitéde ne pas chaufferle lait plus qu'il n'esta bsolument nécessaire.

Comme on le sait, le chauffage d'une culture bactérienne ne détruit pas ensemble toutes les cellules végétatives, mais quelques-unes supportent, ainsi que je l'ai montré en ce qui concerne les bactéries lactiques, tout au moins jusqu'à $15^{\circ} \mathrm{C}$. au-dessus de la température qui détruit la plupart des cellules. Ce qui toutefois a plus d'intérêt à cet égard, c'est que les cellules ainsi survivantes sont généralement moins résistantes et par conséquent meurent facilement quelque temps après la pasteurisation. Nous reviendrons plus loin sur cette question. En ce qui concerne les bactéries pathogènes, elles perdent, comme on le sait, souvent leur virulence par le chauffage, et en ce qui concerne plus spécialement les bactéries de la tubereulose, je rappellerai ici que le Professeur B. BANG a montré que les bactéries de la tuberculose sont déjà tellement ébranlées fortement par 2 minutes de chauffage à $60^{\circ} \mathrm{C}$., qu'elles ne peuvent plus 
TABLEAU XLVI

\begin{tabular}{|c|c|c|c|c|c|c|c|c|c|c|c|c|c|c|}
\hline \multirow{2}{*}{ Chauffage } & \multicolumn{2}{|c|}{ Lait glacé } & \multicolumn{2}{|c|}{ Lait pour enfants $\mathrm{I}$} & \multicolumn{2}{|c|}{ Lait pour enfants 2} & \multicolumn{2}{|c|}{ Lait pour enfants 3} & \multicolumn{2}{|c|}{ Lait I } & \multicolumn{2}{|l|}{ Lait 2} & \multicolumn{2}{|c|}{ Lait . 3} \\
\hline & $\begin{array}{l}\text { Nombre } \\
\text { de germes }\end{array}$ & $\%$ & $\begin{array}{c}\text { Nombre } \\
\text { de germes }\end{array}$ & $\%$ & $\begin{array}{l}\text { Nombre } \\
\text { de germes }\end{array}$ & $\%$ & $\begin{array}{l}\text { Nombre } \\
\text { de germes }\end{array}$ & $\%$ & $\begin{array}{l}\text { Nombre } \\
\text { de germes }\end{array}$ & $\%$ & $\begin{array}{l}\text { Nombre } \\
\text { de germes }\end{array}$ & $\%$ & $\begin{array}{l}\text { Nombre } \\
\text { de germes }\end{array}$ & $\%$ \\
\hline cru & 25.300 & & 27.500 & & 90.000 & & 60.000 & & 160.000 & & 470.000 & & 750.000 & \\
\hline $62^{\circ} \mathrm{C}$. pendant $30 \mathrm{~min}$. & 200 & 8 & 200 & 7 & 400 & 4 & 200 & 3 & 500 & 3 & 2.300 & 5 & 1.500 & 2 \\
\hline $63^{\circ} \mathrm{C}$. pendant $30 \mathrm{~min}$. & 100 & 4 & 100 & 4 & 200 & 2 & 200 & 3 & 200 & 1 & 1.700 & 4 & 900 & 1 \\
\hline
\end{tabular}


TABLEAU XLVII

\begin{tabular}{|c|c|c|c|c|c|c|c|c|c|c|}
\hline Chauffage & $\begin{array}{l}\text { Immédiate- } \\
\text { ment } \\
\text { après pas- } \\
\text { teurisation }\end{array}$ & $\begin{array}{l}\text { Après } \\
24 \text { heures } \\
\text { a } 5^{\circ} \mathrm{C}\end{array}$ & $\begin{array}{c}\text { Immédiate- } \\
\text { ment } \\
\text { après pas- } \\
\text { teurisation }\end{array}$ & $\begin{array}{l}\text { Après } \\
24 \text { heures } \\
\text { à } 5^{\circ} \mathrm{C}\end{array}$ & $\begin{array}{c}\text { Immédiate- } \\
\text { ment } \\
\text { après pas- } \\
\text { teurisation }\end{array}$ & $\begin{array}{l}\text { Après } \\
24 \text { beures } \\
\text { à } 5^{\circ} \mathrm{C}\end{array}$ & $\begin{array}{c}\text { Immédiate- } \\
\text { ment } \\
\text { apress pas- } \\
\text { teurisation }\end{array}$ & $\begin{array}{l}\text { Après } \\
24 \text { heures } \\
\text { à } 5^{\circ} \mathrm{C}\end{array}$ & $\begin{array}{c}\text { Immédiate- } \\
\text { ment } \\
\text { aprés pas- } \\
\text { teurisation }\end{array}$ & $\begin{array}{l}\text { Après } \\
24 \text { heures } \\
\text { a } 5^{\circ} \mathrm{C}\end{array}$ \\
\hline & \multicolumn{2}{|c|}{ Lait du soir 1} & \multicolumn{2}{|c|}{ Lait du soir 2} & \multicolumn{2}{|c|}{ Lait du soir 3} & \multicolumn{2}{|c|}{ Lait du soir 4} & \multicolumn{2}{|c|}{ Lait du soir 5} \\
\hline \multirow{3}{*}{$\begin{array}{l}\text { cru } \\
63^{\circ} \text { C. pendant } 30 \text { minutes } \\
68^{\circ}\end{array}$} & 77.700 & 59.110 & 270.100 & 156.000 & 145.600 & 113.400 & 257.500 & 132.300 & 135.100 & 114.200 \\
\hline & 700 & 170 & 300 & 100 & 440 & 290 & 200 & 90 & 910 & 430 \\
\hline & 700 & 120 & 200 & 40 & 500 & 160 & 100 & 36 & 890 & 390 \\
\hline & \multicolumn{2}{|c|}{ Lait du soir 6} & \multicolumn{2}{|c|}{ Lait du soir 7} & \multicolumn{2}{|c|}{ Lait du soir 8} & \multicolumn{2}{|c|}{ Lait du soir 9} & \multicolumn{2}{|c|}{ Lait du soir 10} \\
\hline \multirow{3}{*}{ 63० C. pendant 30 minutes } & 13.400 & 10.800 & 19.600 & 7.600 & 34.700 & 25.100 & 32.500 & 11.340 & 43.800 & 580.000 \\
\hline & 190 & 100 & 180 & 110 & 590 & 480 & 280 & 170 & 170 & 40 \\
\hline & 240 & 100 & 200 & 90 & 240 & 200 & 330 & 200 & 90 & 50 \\
\hline & \multicolumn{2}{|c|}{ Lait du soir 11} & \multicolumn{2}{|c|}{ Lait du soir 12} & \multicolumn{2}{|c|}{ Lait du soir 13} & \multicolumn{2}{|c|}{ Lait du matin 14} & \multicolumn{2}{|c|}{ Lait du matin 15} \\
\hline \multirow{2}{*}{ 630 } & 35.600 & 100.000 & 80.000 & 1.110 .000 & 37.200 & 210.000 & 90.000 & 107.100 & 56.000 & 50.000 \\
\hline & 100 & 50 & 9.400 & 5.440 & 5.800 & 2.890 & 490 & 230 & 380 & 120 \\
\hline $68^{\circ} n$ n 5 & 40 & 30 & 8.400 & 4.730 & 9.000 & 3.920 & 420 & 300 & 250 & 110 \\
\hline & \multicolumn{2}{|c|}{ Lait du matin 16} & \multicolumn{2}{|c|}{ Lait du matin 17 । } & \multicolumn{2}{|c|}{ Lait du matin 18} & \multicolumn{2}{|c|}{ Lait du matin 19} & \multicolumn{2}{|c|}{ Lait du matin 20} \\
\hline cru & 22.200 & 28.700 & 130.000 & 270.000 & 29.400 & 27.500 & 7.600 & 7.100 & 23.800 & 22.900 \\
\hline \multirow{3}{*}{$\begin{array}{l}63^{\circ} \mathrm{C} \text {. pendant } 30 \text { minutes } \\
68^{\circ}\end{array}$} & 200 & 120 & 2.110 & 430 & 60 & 20 & 10 & & 30 & 20 \\
\hline & 460 & 90 & 1.300 & 410 & 30 & 12 & 20 & 8 & 20 & 12 \\
\hline & \multicolumn{2}{|c|}{ Lait du matin 21} & \multicolumn{2}{|c|}{ Lait du matin 22} & \multicolumn{2}{|c|}{ | Lait du matin 23} & Lait du n & matin 24 & Lait du & matin 25 \\
\hline eru & 13.800 & 11.600 & 250.000 & 280.000 & 1.690 .000 & 3.310 .000 & 200.000 & 180.000 & 190.000 & $\overline{160.000}$ \\
\hline $63^{\circ} \mathrm{C}$. pendant 30 minutes & 30 & 10 & 20 & & 120 & 50 & 20 & & 350 & 220 \\
\hline $68^{\circ}$ " $\quad 5 \quad$ & 20 & 6 & 20 & & 80 & 60 & 40 & 6 & 400 & 290 \\
\hline & Lait du $n$ & matin 26 & Lait du m & natin 27 & Lait du m & matin 28 & Lait du n & matin 29 & Lait du & matin 30 \\
\hline eru & 210.000 & 120.000 & 340.000 & 540.000 & 310.000 & 790.000 & 290.000 & 250.000 & 80.000 & 110.000 \\
\hline $63^{\circ} \mathrm{C}$. pendant 30 minutes & 1.200 & 910 & 8.060 & 6.930 & 280 & 270 & 300 & 120 & 90 & 46 \\
\hline $68^{\circ} \infty \quad 5 \quad 5$ & 1.200 & 1.080 & 7.470 & 6.560 & 300 & 200 & 300 & 180 & 100 & 60 \\
\hline & Lait du $\mathrm{r}$ & matin 31 & Lait du $\mathrm{m}$ & natin 32 & Lait du $\mathrm{m}$ & matin 33 & Lait du $\mathrm{n}$ & matin 34 & Lait du & matin 35 \\
\hline cru & 130.000 & 350.000 & 43.400 & 53.300 & 650.000 & 567.000 & 5.540 .000 & 10.030 .000 & 6.780 .000 & 11.340 .000 \\
\hline $63^{\circ} \mathrm{C}$. pendant 30 minutes & 90 & & 1.200 & 1.480 & 100 & 74 & 330 & 210 & 140 & 70 \\
\hline $68^{\circ} \times 2$ & 90 & 56 & 1.700 & 2.100 & 100 & 54 & 200 & 150 & 120 & 70 \\
\hline & Lait du $n$ & matin 36 & Lait du $\mathrm{m}$ & natin 37 & Lait du $\mathrm{m}$ & matin 38 & Lait du n & matin 39 & Lait du & matin 40 \\
\hline cru & $7.380 .000 \mid$ & 17.800 .000 & 2.640 .000 & 7.200 .000 & 72.000 & 110,000 & 210.000 & 70.000 & 630.000 & 1.080 .000 \\
\hline $63^{\circ} \mathrm{C}$. pendant 30 minutes & 24.000 & 10.080 & 490 & 320 & 60 & 20 & 910 & 560 & 80 & 40 \\
\hline 680 & 20.600 & 7.560 & 370 & 340 & 10l & & 710 ا & & & \\
\hline
\end{tabular}


occasionner la tuberculose par l'alimentation, et il n'y a que cela qui soit intéressant en ce qui concerne le lait du commerce, qui normalement n'est pas employé sous la forme d'injections sous-cutanées.

Le Professeur O. BANG a confirmé par quelques recherehes, qu'il a bien voulu renouveler pour moi récemment, que la virulence des bactéries de la tuberculose est ainsi ébranlée par le chauffage, avant qu'elles ne soient détruites, et je l'en remercie ici. Ces recherches ont montré que les bactéries du lait de vaches ayant la tuberculose du pis sont toutes détruites quand le lait est chauffé 5 minutes à $68^{\circ} \mathrm{C}$. Les cobayes inoculés avec le même lait à l'état cru moururent tous de tuberculose généralisée en 2 mois. En même temps, on inocula une paire de cobayes avec le même lait chauffé 5 minutes à 5904-59 $7 \mathrm{C}$. Ces animaux moururent aussi de tuberculose généralisée, mais seulement après respectivement 5 et 6 mois. Le dit chauffage avait ainsi diminué considérablement la virulence des bacilles de la tubereulose.

Nous arrivons maintenant à une propriété du lait qui n'est pas sans intérêt pour la question de la pasteurisation : e'est celle de son pouvoir bactéricide.

Jusqu'à ce que j'ai découvert (1) en 1915, à l'occasion de quelques recherches de la biorisation que le pouvoir bactéricide du lait n'est pas détruit par la pasteurisation basse, la plupart des chercheurs le considéraient comme une propriété qui disparaissait peu de temps après la traite du lait. La cause en était que la propagation des bactéries du lait - et aussi celle des bactéries qui peuvent se développer à basse température - est déjá tellement bien en train peu de temps après la traite que, même si le lait à partir de ce moment est conservé à basse température, elle contre-balanse l'arrêt ou les destructions que le pouvoir bactéricide produit. Autrement, si le lait, avant qu'il ne soit placé à basse température, a été chauffé à une température qui, sans détruire son pouvoir bactéricide tue la plupart de ses bactéries et spécialement les bactéries qui peuvent se développer à basse température, on observera généralement dans ces conditions une diminution bactérienne évidente dans le lait. La température de $5^{\circ} \mathrm{C}$. semble ici être la température de conservation la plus favorable, parce que, généralement, elle est suffisante pour freiner la multiplication des bactéries sans arrêter entièrement l'action du pouvoir bactéricide du lait.

Dans le tableau XLVII, dont il a déjà été questionà un autre point de vue, on voit l'action du pouvoir bactériciae dans le lait de 40 vaches différentes. Le lait du matin était au moment de l'examen trait depuis 3 à 4 heures, et le lait du soir depuis 15 à 16 heures. Comme au moment où les examens avaient lieu le temps était froid, le lait n'était refroidi qu'à l'air.

(1) Moelkeritidende, 1915, p. 483. 
Comme on le voit, il y a dans le lait ayant subi la pasteurisation basse une diminution visible de bactéries quand on le laisse 24 heures à $5^{\circ} \mathrm{C}$. Il n'y a que dans le lait du matin No 32 qu'on n'ait pas observé de diminution de bactéries, mais on n'a pas non plus constatéde multiplication appréciable de bactéries. La différence dans les nombres de germes n'est pas plus grande qu'une erreur possible dans la numération des germes. Il en est autrement dans le lait à l'état non pasteurisé. Ici, nous avons environ $1 / 3$ des échantillons de lait avec une augmentation de bactéries évidente et cela naturellement de préference cans les échantillons les plus riches en bactéries.

La question n'est pas toujours aussi simple avec le lait du commerce, qui peut aussi de temps en temps montrer une augmentation bactérienne minime, quand on le conserve à $5^{\circ} \mathrm{C}$. a près la pasteurisation basse. Ceci est le cas du lait qui contient beaucoup de spores bactériennes en plus des tétracoques (microcoques) et des microbactéries, qui ne sont pas du tout touchées par la pasteurisation brsse. Des essais spéciaux avec ensemencement de diverses sortes de bactéries dans du lait cru, pauvre en bactéries et avec ensuite pasteurisation basse, ont montré que pendant le repos à $5^{\circ} \mathrm{C}$., ce sont spécialement les bactéries qui ont le plus souffert de la pasteurisation lente (par exemple les colibacteries) que le pouvoir bactéricide du lait maîtrise.

Des tableaux XLVIII et XLIX, on voit que le pouvoir bactericide du lait n'est détruit que par un chauffage de quelques minutes à $70^{\circ}$ $75^{\circ} \mathrm{C}$. Il n'est généralement pas, comme d'autres essais nous l'ont montré, détruit dans du lait traité par le procédé Stassano (lait instantanement chauffé à $75^{\circ} \mathrm{C}$.), et d'après les essais a ctuels, il semble pouvoir supporter un chauffage à $68^{\circ} \mathrm{C}$. pendant 30 minutes. Si nous étudions de plus près le lait qui n'a été chauffé que 30 minutes à $63^{\circ} \mathrm{C}$. ou 5 mirutes à $68^{\circ} \mathrm{C}$., le tableau XLVIII montre que si un tel lait est conservé à $5^{\circ} \mathrm{C}$., la diminution des bactéries n'est pas à son point culminant a près 12 heures, et le tableau XLIX montre qu'elle est par contre maximum après 24 heures, ou en tout cas avant qu'il ne se soit écoulé 48 heures. Il s'ensuit que la conservation, pendant quelque temps, du lait ayant subi la pasteurisation basse, ce qui, du reste, est inévitable dans la fourniture des grandes villes, n'abîme pas du tout le lait, mais le rend au contraire encore plus pauvre en germes, et même le débarrasse peut-être de bactéries de maladies encore survivantes, mais très ébranlées. La seule condition est que la conservation ait lieu à basse température.

Nous n'avons pas réussi à determiner ce que signifie exactement le pouvoir bactéricide du lait, ou ce qui plus exactement devrait être appelé les pouvoirs bactéricides. Qu'il ne soit pas lié entièrement à l'agglutinine ressort de ce que l'agglutinine est déjà détruite par 5 minutes de chauffage à $68^{\circ} \mathrm{C}$. tandis que le lait se montre précisément fortement bactéricide a près ce traitement. Le pouvoir bactérieide du lait 
TABLEAU XLVIII

\begin{tabular}{|c|c|c|c|c|c|c|c|c|c|c|c|c|c|c|}
\hline \multirow{3}{*}{\multicolumn{3}{|c|}{ Chauffage }} & \multicolumn{6}{|c|}{ Lait 1} & \multicolumn{6}{|c|}{ Lait pour enfants I } \\
\hline & & & \multicolumn{2}{|c|}{$\begin{array}{l}\text { Immédiatement après } \\
\text { pasteurisation }\end{array}$} & \multicolumn{2}{|c|}{$\begin{array}{l}\text { Après } 12 \text { heures } \\
\text { à } 5^{\circ} \mathrm{C}\end{array}$} & \multicolumn{2}{|c|}{$\begin{array}{l}\text { Après } 24 \text { heures } \\
\text { à } 5^{\circ} \mathrm{C}\end{array}$} & \multicolumn{2}{|c|}{$\begin{array}{l}\text { Immédiatement après } \\
\text { pasteurisation }\end{array}$} & \multicolumn{2}{|c|}{$\begin{array}{c}\text { A près } 12 \text { heures } \\
\text { à } 5^{\circ} \mathrm{C}\end{array}$} & \multicolumn{2}{|c|}{$\begin{array}{c}\text { Après } 24 \text { heures } \\
\text { à } 5^{\circ} \mathrm{C}\end{array}$} \\
\hline & & & $\begin{array}{l}\text { Nombre } \\
\text { de germes }\end{array}$ & $\%$ & $\begin{array}{l}\text { Nombre } \\
\text { de germes }\end{array}$ & $\%$ & $\begin{array}{l}\text { Nombre } \\
\text { de germes }\end{array}$ & $\%$ & $\begin{array}{l}\text { Nombre } \\
\text { de germes }\end{array}$ & $\%$ & $\begin{array}{c}\text { Nombre } \\
\text { de germes }\end{array}$ & $\%$ & $\begin{array}{l}\text { Nombre } \\
\text { de germes }\end{array}$ & $\%$ \\
\hline . & cru & & 5.420 .000 & & & & 6.830 .000 & & 1.010 .000 & & & & 320.000 & 316 \\
\hline $63^{\circ} \mathrm{C}$ & pendant 30 & minutes & 5.100 & 1 & 1.400 & 0,3 & 900 & 0,2 & 2.700 & 2 & 600 & 0,6 & 350 & 0,3 \\
\hline $68^{\circ}$, & $\Rightarrow \quad 5$ & 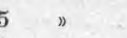 & 13.300 & 3 & 3.900 & ] & 2.100 & 0,4 & 2.500 & 2 & 1.100 & 1 & 840 & 0,8 \\
\hline D & 1) 1 & 150 & 4.500 & 1 & 2.650 & 0,5 & 1.700 & 0,3 & 1.800 & 2 & -700 & 0,7 & 400 & 0,4 \\
\hline ") & , 3 & 》) & 2.500 & 0,5 & 1.200 & 0,2 & 1.600 & 0,3 & 1.400 & 1 & 500 & 0,5 & 900 & 0,9 \\
\hline $70^{\circ}$ & 3 & $n$ & 12.900 & 2 & 12.000 & 2 & 13.000 & 2 & 3.000 & 3 & 2.900 & 3 & 3.600 & 3 \\
\hline$n$ & ") & 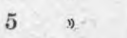 & 4.000 & 1 & & & 5.100 & 1 & 1.200 & 1 & & & 1.900 & 2 \\
\hline $75^{\circ}$ & 》) & 11 & 1. 700 & 0,3 & & & 3.850 & 1 & 600 & 0,6 & & & 1.500 & 1 \\
\hline , & 0 & $2 》$ & 1.150 & 0,2 & & - & 2.100 & 0,4 & 320 & 0,3 & & s & 1.100 & 1 \\
\hline$n$ & $n \quad y=$ & $5-7$ & 660 & 0,1 & & & 1.100 & 0,2 & 150 & 0,1 & & & 400 & 0,4 \\
\hline $80^{\circ}$ & " instantané & ément & 700 & 0,1 & & & 1.600 & 0,3 & 380 & 0,4 & & & 1.400 & 1 \\
\hline & Dendant & 2 minutes & 320 & 0,1 & & & 1.400 & 0,3 & 280 & 0,3 & & & 1.300 & 1 \\
\hline $85^{\circ}$ & ") instantané & ément & 250 & 0,05 & & & 900 & 0,2 & 110 & 0,1 & & & 400 & 0,4 \\
\hline$n$ & "pendant & 2 minutes & 160 & 0,03 & & & 500 & 0,1 & 60 & 0,06 & & & 200 & 0,2 \\
\hline
\end{tabular}


TABLEAU XLIX

\begin{tabular}{|c|c|c|c|c|c|c|c|c|c|c|c|c|c|c|c|}
\hline \multirow{3}{*}{\multicolumn{4}{|c|}{ Chauffage }} & \multicolumn{6}{|c|}{ Lait glacé } & \multicolumn{6}{|c|}{ Lait pour enfants I } \\
\hline & & & & \multicolumn{2}{|c|}{$\begin{array}{l}\text { Immédiatement } \\
\text { après pasteurisation }\end{array}$} & \multicolumn{2}{|c|}{$\begin{array}{c}\text { Après } 24 \text { heures } \\
\text { à } 5^{\circ} \mathrm{C}\end{array}$} & \multicolumn{2}{|c|}{$\begin{array}{c}\text { Après } 48 \text { heures } \\
\text { à } 5^{\circ} \mathrm{C}\end{array}$} & \multicolumn{2}{|c|}{$\begin{array}{l}\text { Immédiatement } \\
\text { après pasteurisation }\end{array}$} & \multicolumn{2}{|c|}{$\begin{array}{c}\text { Après } 24 \text { heures } \\
\text { à } 5^{\circ} \mathrm{C}\end{array}$} & \multicolumn{2}{|c|}{$\begin{array}{l}\text { Après } 4^{8} \text { heures } \\
\text { à } 5^{\circ} \mathrm{C}\end{array}$} \\
\hline & & & & $\begin{array}{l}\text { Nombres } \\
\text { de germes }\end{array}$ & $\%$ & $\begin{array}{c}\text { Nombre } \\
\text { de germes }\end{array}$ & $\%$ & $\begin{array}{l}\text { Nombre } \\
\text { de germ. }\end{array}$ & $\%$ & $\begin{array}{l}\text { Nombre } \\
\text { de germes }\end{array}$ & $\%$ & $\begin{array}{l}\text { Nombre } \\
\text { de germes }\end{array}$ & $\%$ & $\begin{array}{l}\text { Nombre } \\
\text { de germes }\end{array}$ & $\%$ \\
\hline & & & & 23.600 & & 5.100 & 216 & & & 730.000 & & 520.000 & 712 & & \\
\hline $63^{\circ} \mathrm{C}$. & nde & 30 & inutes & 600 & 25 & 50 & 2 & 200 & 9 & 2.400 & 3 & 400 & 1 & 600 & 1 \\
\hline $68^{\circ} "$ & » & 5 & , & 300 & 13 & 40 & 2 & 100 & 4 & 1.200 & 2 & 100 & 0,2 & 600 & 1 \\
\hline $70^{\circ}$ ” & 》 & 5 & 》 & $200^{\circ}$ & 9 & 300 & 13 & 400 & 17 & 900 & 1 & 1.100 & 2 & 3.500 & 5 \\
\hline $75^{\circ}$ 》 & , & 5 & $"$ & 30 & 1 & 100 & 4 & 200 & 9 & 200 & 0,5 & 500 & 1 & 800 & 1 \\
\hline \multicolumn{4}{|c|}{$80^{\circ}$ instantanément } & 100 & 4 & 100 & 4 & 300 & 13 & 500 & 1 & 400 & 1 & 900 & 1 \\
\hline
\end{tabular}


ne semble pas non plus à un degré notable être lié aux leucocytes du lait, car, d'un côté, il ne diminue pas par la purification centrifuge du lait, et, d'un autre eôté, il n'augmente pas non plus, même si nous augmentons considérablement la teneur en leucocytes au lait en l'additionnant de 100 fois d'autant de boue fraîche de centrifuge que le lait en sépare normalement par la centrifugation. La diminution des bactéries lors de la conservaticn du lait ayant été soumis à la pasteurisation basse semblait même dans ces cireonstances être plus minime, ce qui peut provenir peut-être de la grande quantité de bactéries qu'on ajoute au lait avec la boue. Dans quelques cas, le lait additionné de boue a montré par contre une petite diminution de bactéries quand il était conservé après avoir été chauffé à $80^{\circ} \mathrm{C}$., de même qu'un tel lait n'était pas toujours entièrement négatif à la réaction Sтовсн. Ceci montre que la boue protège quelque peu contre la chaleur (1).

Comme je l'ai déjà dit, les leucocytes du lait perdent petit à petit leur propriété de réduire le bleu de méthylène quand le lait est chauffé ì plus de $70^{\circ} \mathrm{C}$. Il était done intéressant, en liaison avec la question analogue traitée, de voir de plus près comment le pouvoir bactéricide du lait se tenait à cette température, puısque nous savons déjà que celui-ci a presque les mêmes limites de température que les leucocytes. Malheureusement, les essais résumés dans le tableau $L$ n'ont pas donné d'indication précise, car les échantillons de lait employés se montrèrent être extraordinairement peu bactéricides. Nous rendons néanmoins compte de ces essais, parce qu'à cette occasion nous avons aussi déterminé la durée de coagulation du lait (exprimé en jours) à $22^{\circ} \mathrm{C}$. à la fois immédiatement après la pasteurisation et après les 24 heures de repos à $5^{\circ} \mathrm{C}$. Cette durée de coagulation est presque une mesure de la force vitale des bactéries lactiques et des bacilles du foin existant dans le lait. On voit maintenant que la conservation du lait est augmentée quand celui-ci reste 24 heures après la pasteurisation à $5^{\circ} \mathrm{C}$.; mais ce qui est remarquable, c'est que ceci est le plus souvent également exact, aussi bien pour le lait ayant subi la pasteurisation haute que pour celui ayant subi la pasteurisation basse. Le pouvoir bactéricide du lait ne semble ainsi pas entièrement détruit dans le lait qui a été chauffé à $80^{\circ} \mathrm{C}$., même si généralement dans un tel lait il n'est plus en mesure de détruire les bactéries.

Nous avons en réalité montré que le pouvoir bactéricide du lait se compose de divers composants, mais nous conservons ces recherches pour un travail spécial.

J'ai montré,il y a déjà desannées (voirma bactériologielaitière)qu'il peut se produire dansle lait placéà basse température une augmentation active

(1) Dans les bains de boue, où la boue se compose souvent de terre d'infusoires ou d'au. tres produits chimiquement inactifs, la seule action thérapeutique de la boue est proba blement qu'elle permet aux patients de supporter une plusforte température qu'ils ne le peuvent dans l'eau pure. 
TAB̈LEAU L

\begin{tabular}{|c|c|c|c|c|c|c|c|c|c|c|c|c|c|c|c|c|c|c|}
\hline & \multirow{3}{*}{ Chauffage } & \multirow{3}{*}{$m$} & \multicolumn{4}{|c|}{ Lait pour enfants 2} & \multicolumn{4}{|c|}{ Lait 2} & \multicolumn{4}{|c|}{ Lait pour enfants I } & \multicolumn{4}{|c|}{ Lait $\mathrm{I}$} \\
\hline & & & \multicolumn{2}{|c|}{$\begin{array}{c}\text { Immédiatement } \\
\text { après } \\
\text { pasteurisation }\end{array}$} & \multicolumn{2}{|c|}{$\begin{array}{c}\text { Après } 24 \text { heures } \\
\text { à } 5^{\circ} \mathrm{C}\end{array}$} & \multicolumn{2}{|c|}{$\begin{array}{c}\text { Immédiatement } \\
\text { après } \\
\text { pasteurisation }\end{array}$} & \multicolumn{2}{|c|}{$\begin{array}{c}\text { Après } 24 \text { heures } \\
\text { à } 5^{\circ} \mathrm{C}\end{array}$} & \multicolumn{2}{|c|}{$\begin{array}{c}\text { Immédiat. } \\
\text { après } \\
\text { pasteurisation }\end{array}$} & \multicolumn{2}{|c|}{$\begin{array}{c}\text { Après } 24 \text { heures } \\
\text { à } 5^{\circ} \mathrm{C}\end{array}$} & \multicolumn{2}{|c|}{$\begin{array}{l}\text { Immédiatement } \\
\text { après } \\
\text { pasteurisation }\end{array}$} & \multicolumn{2}{|c|}{$\begin{array}{l}\text { Après } 24 \text { teures } \\
\text { à } 5^{\circ} \mathrm{C}\end{array}$} \\
\hline & & & $\begin{array}{c}\text { Nombre } \\
\text { de } \\
\text { germes }\end{array}$ & $\begin{array}{c}\text { Durée } \\
\text { de } \\
\text { coagul. }\end{array}$ & $\begin{array}{c}\text { Nombre } \\
\text { de } \\
\text { germes }\end{array}$ & $\begin{array}{c}\text { Dur. } \\
\text { de } \\
\text { coag. }\end{array}$ & $\left|\begin{array}{c}\text { Nombre } \\
\text { de } \\
\text { germes }\end{array}\right|$ & $\begin{array}{c}\text { Durée } \\
\text { de } \\
\text { coag. }\end{array}$ & $\begin{array}{c}\text { Nombre } \\
\text { de } \\
\text { germes }\end{array}$ & $\begin{array}{c}\text { Dur. } \\
\text { de } \\
\text { coag. }\end{array}$ & $\begin{array}{c}\text { Nombre } \\
\text { de } \\
\text { germes }\end{array}$ & $\left|\begin{array}{c}\text { Durée } \\
\text { de } \\
\text { coag. }\end{array}\right|$ & $\begin{array}{c}\text { Nombre } \\
\text { de } \\
\text { germes }\end{array}$ & $\begin{array}{c}\text { Dur. } \\
\text { de } \\
\text { coag. }\end{array}$ & $\begin{array}{l}\begin{array}{c}\text { Nombre } \\
\text { de } \\
\text { germes }\end{array}\end{array}$ & $\begin{array}{c}\text { Dur. } \\
\text { de } \\
\text { coag. }\end{array}$ & $\begin{array}{c}\text { Nombre } \\
\text { de } \\
\text { germes }\end{array}$ & $\begin{array}{c}\text { Durée } \\
\text { de } \\
\text { coag. }\end{array}$ \\
\hline & cru & & 190.000 & $\begin{array}{ll}1 & 1 / 2\end{array}$ & 490.000 & 2 & 150.000 & $11 / 4$ & 2.000 .000 & 3 & 27.400 & $1 \quad 1 / 4$ & 600.000 & 3 & 7.000 .000 & 1 & 37.000 .000 & 2 \\
\hline $63^{\circ} \mathrm{C}$ & C. pendant 30 & $0 \mathrm{~min}$. & 4.700 & 5 & & 7 & & & & & & & & & & & & \\
\hline $68^{\circ}$ & 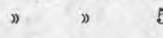 & $5 "$ & 4.100 & 4 & 4.200 & 4 & 14.700 & 4 & 9.800 & 5 & 7.500 & 2 & 6.500 & 3 & 9.400 & 2 & 8.600 & 3 \\
\hline $69^{\circ}$ & 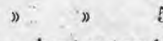 & $5 \leadsto$ & 2.600 & 4 & 2.100 & 4 & 13.000 & 3 & 10.200 & 3 & $6.600 \mid$ & 2 & 5.200 & 3 & 7.600 & 2 & 7.000 & 3 \\
\hline $70^{\circ}$ & " instantané & & 16.700 & 3 & 15.100 & 7 & 14.900 & 3 & 14.400 & 3 & 12.000 & 2 & 11.700 & 3 & 25.000 & 2 & 23.700 & 3 \\
\hline 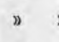 & "pendant & $1 \mathrm{~min}$. & & & & & 13.600 & 2 & 13.200 & 3 & 14.400 & 3 & 11.200 & 3 & 16.000 & 2 & 15.900 & 3 \\
\hline ” & $\Rightarrow \quad \Rightarrow=$ & $2 "$ & & & & & 13.100 & 2 & 12.900 & 4 & 9.700 & 3 & 9.600 & 3 & 10.400 & 2 & 11.500 & 3 \\
\hline$"$ & \# & $5 \gg$ & 22.00 & 5 & 3.000 & 8 & -11.100 & 3 & 11.100 & 4 & 6.200 & 2 & 6.300 & 3 & 7.400 & 2 & 9.500 & 3 \\
\hline $75^{\circ}$ & 1) instantané & & 2.300 & 3 & 4.500 & 3 & 11.000 & 2 & 11.200 & 3 & 9.700 & 2 & 9.700 & 3 & 10.200 & 2 & 11.000 & 3 \\
\hline$”$ & "pendant 1 & 1 min. & & & & & 8.800 & 2 & 9.009 & 3 & 9.100 & 2 & 9.100 & 3 & 9.600 & 2 & 9.900 & 3 \\
\hline 80 & " instantané & & 900 & 3 & 1.500 & 3 & 9.400 & 2 & 9.600 & 3 & 8.700 & 2 & 9.100 & 3 & 7.200 & 2 & 7.900 & 3 \\
\hline & "pendant & $1 \mathrm{~min}$. & & & & & 6.200 & 2 & 6.300 & 3 & 5.200 & 2 & 5.400 & 3 & 6.200 & 2 & 6.400 & 3 \\
\hline
\end{tabular}


de bactéries liquéfiant la gelatine, même si le nombre total des germes diminue. Le tableau LI montre maintenant inversement que même si le lait cru qui est conservé au froid montre une augmentation nette de bactéries (une augmentation de bactéries psychrophiles), il se produit tout de même dans ce lait une destruction importante des bactéries originales. Après pasteurisation, ce lait conservé à $5^{\circ} \mathrm{C}$. contenait en effet - même après 3 fois 24 heures - moins de bactéries que ne contenait le même lait pasteurisé avant le rèpos. Même le dernier lait (lait pour enfants 1) etait plus pauvre en germes après pasteurisation quand il était resté 3 fois 24 heures à $10^{\circ} \mathrm{C}$. que quand il etait pasteurisé immédiatement. Ceci n'est naturellement pas synonyme de meilleure qualité, car la saveur désagréable que produisent les bactéries fluorescentes, ne disparaît pas par la pasteurisation.

\section{TABLEAU LI}

\begin{tabular}{|c|c|c|c|c|c|c|c|c|c|c|}
\hline \multirow{2}{*}{\multicolumn{5}{|c|}{$\begin{array}{c}\text { Conservation } \\
\text { avant pasteurisation }\end{array}$}} & \multicolumn{2}{|c|}{ Lait 2} & \multicolumn{2}{|c|}{ Lait pour enfants 2} & \multicolumn{2}{|c|}{ Lait pour enfants $x$} \\
\hline & & & & & cru & $68^{\circ} \mathrm{C}$ p. $5 \mathrm{~min}$. & cru & $68^{\circ} \mathrm{C}$ p. $5 \mathrm{~min}$. & $\mathrm{cru}$ & $68^{\circ} \mathrm{C}$ p. $5 \mathrm{~min}$. \\
\hline \multirow{2}{*}{\multicolumn{5}{|c|}{$\begin{array}{l}\text { Immédiatement } \\
5^{\circ} \mathrm{C} \text {. pendant } 24 \text { heures }\end{array}$}} & 930.000 & 4. 000 & 230.000 & 3.000 & 610.000 & 11.000 \\
\hline & & & & & 1.010 .000 & 3.900 & 190.000 & 1.900 & 2.000 .000 & 2. 600 \\
\hline 0 & & d) 4 & 48 & (1) & 2.000 .000 & 1. 600 & 500.000 & 1.800 & 12.000 .000 & 3.300 \\
\hline x & $a$ & ग & 72 & 》 & 85.000 .000 & 1.900 & 7.000 .000 & 2.100 & 87.000 .000 & 3.900 \\
\hline $10^{\circ}$ & x & 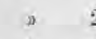 & 24 & n & 1.300 .000 & 4.100 & 410.000 & 2.000 & 12.000 .000 & 2.600 \\
\hline$\Rightarrow$ & $x$ & is & 48 & $\Rightarrow$ & 25.000 .000 & 4.300 & 8.000 .000 & 3.600 & 72.000 .000 & 3.900 \\
\hline B & $x$ & n & 72 & $n$ & 236.000 .000 & 5.600 & 96.000 .000 & $3: 900$ & 331.000 .000 & 4. 600 \\
\hline
\end{tabular}

Dans ces essais, où le lait devait être conservé longtemps à l'état cru, on ne pouvait pas employer une température de conservation plus élevée que $10^{\circ} \mathrm{C}$., car autrement on risquait que le lait ne se coagule avant ou en tout cas pendant la pasteurisation qui suivait. Au contraire, lors des essais résumés dans le tableau LII, où nous conservions le lait à l'état pasteurisé, on a très bien pu employer une température de conservation allant jusqu'à $20^{\circ} \mathrm{C}$.

Ce tableau montre que quand le lait ayant subi la pasteurisation lente, au lieu d'être conservé à $5^{\circ} \mathrm{C}$., l'est à $15^{\circ} \mathrm{C}$. ou $20^{\circ} \mathrm{C}$., une rapide augmentation de bactéries a déjà lieu au cours des premières 24 heures, mais si on conserve le lait 24 heures de plus - bien noter que c'est à $5^{\circ} \mathrm{C}$. - le nombre des bactéries diminue à nouveau. On voit que le pouvoir bactéricide du lait n'est pas encore détruit dans le lait pasteurisé, après que celui-ci a été conservé 48 heures à $15^{\circ} \mathrm{C}$. ou 24 heurés à $20^{\circ} \mathrm{C}$. Dans tous les cas, nos essais indiquent que le pouvoir bactéricide du lait n'a pas un caractère spécialement instable. 
TABLEAU LII

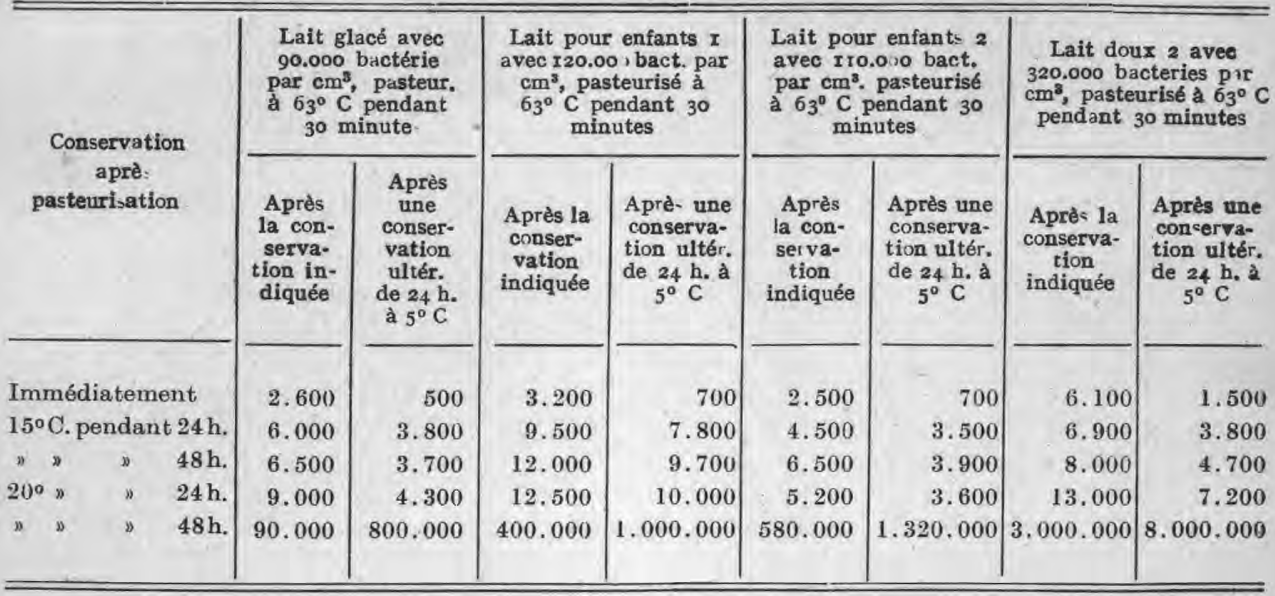

RÉSUMÉ.

1. - Le travail actuel est un complément de recherches antérieures relatives aux modifications physiques, chimiques et bactériologiques qui ont lieu dans le lait soumis à un chauffage court ou prolongé à des températures diverses. On a spécialement étudié la possibilité d'employer des méthodes de pasteurisation basse plus rapides (telle que 5 minutes de chauffage à $68^{\circ}$ C.) au lieu de la pasteurisation lente généralement employée ( 30 minutes de chauffage à $63^{\circ} \mathrm{C}$.) ; mais avant que ces procédés n'aient été utilisés pratiquement, nous n'osons pas recommander de méthodè nouvelle.

2. - Nous avons réussi à montrer que l'albumine du lait qui se coagule entièrement à des températures entre $80^{\circ}$ et $90^{\circ} \mathrm{C}$., se solubilise à nouveau à des températures plus élevées sous forme d'albuminate. Sous cette forme, l'albumine se trouve aussi dans le lait cuit. La coagulation de-l'albumine ainsi que sa nouvelle dissolution, ont lieu dans le sérum à des températures un pəu inférieures à celles auxquelles elles ont lieu dans le lait.

3. - Nous avons établi une méthode plus précise pour la détermination de la proportion d'amylase du lait.

4. - La partie la plus importante de notre étude a été la recherche d'une réaction montrant si le lait a été soumis à la pasteurisation lente, et nous avons réussi à trouver une telle réaction, basée sur la diminution de la montée de la crème que le lait subit par la chaleur; en outre, nous avons étudié particulièrement tous les facteurs qui agissent sur la montée de la erème du lait.

5. - La réaction crémométrique trouvée repose sur ce que la montée de la crème est facilitée dans le lait cru, mais retardée dans le lait ayant 
été soumis à la pasteurisation lente par addition d'eau. Le rapport entre la couche de crème dans le lait étendu et le lait non étendu (calculé sur la même quantité de lait) est par conséquent plus grand que $l$ dans le lait cru, mais inférieur à 1 dans̀ le lait ayant été soumis à la pasteurisation lente. Par une dilution convenable avec de l'eau, on peut obtenir que le changement de rapport corresponde exactement au mode de chauffage employé pour la pasteurisation lente.

6. - La réaction s'exécute de la manière suivante : "Le lait qui doit être étudié est d'abord chauffé à $50^{\circ} \mathrm{C}$. pendant 5 minutes, puis on en aspire avec une pipette respectivement 5 et $10 \mathrm{cmc}$. qu'on transvide dans deux crémo-butyromètres de HoYBERG. Les $5 \mathrm{cmc}$. de lait sont mélangés avec soin avec autant d'eau, et les deux échantillons de lait sont placés pendant deux heures dans de l'eau courante à $12^{\circ}-15^{\circ} \mathrm{C}$. : on note ensuite les épaisseurs des couches de crème." "

7. - Lo traitemant préliminaire sus-indiqué du lait (5 minutes de chauffage à $50^{\circ} \mathrm{C}$.) est nécessaire pour faire disparaître la paresse de la montée de la crème dûe au froid. Les causes de cette paresse de la montée de la crème dûe au iroid et de celle dûe à l'individualité de la vache sont discutées en détail.

8. - Il est montré que le facteur principal de la montée spontanée de la crème du lait est une agglutinine, qui fait se réunir en masses compactes les globules de matière grasse, ce qui fait qu'ils montent beaucoup plus rapidement qu'ils ne le font quand ils sont isolés. Cette agglutinine commence déjà à être détruite après $1 / 2$ heure de chauffage à $63^{\circ} \mathrm{C}$. et est presque entièrement détruite par 5 minutes de chauffage à $68^{\circ} \mathrm{C}$.

9. - L'agglutinine passe dans le sérum du lait et se précipite a vec la fraction de globuline. Pour agir sur les globules gras, il faut toutefois encore un facteur qui passe aussi dans le sérum, mais qui ne se précipite pas a vec la fraction de globuline ou d'albumine. Ce facteur est plus résis. tant à la chaleur que l'agglutinine, car il n'est détruit qu'à des températures supérieures à $90^{\circ} \mathrm{C}$. Donc, puisque l'agglutination exige uncomplément, ce phénomène est peut-être à considérer comme une conglutination.

10. - Le colostrum contient 5 à 10 fois autant d'agglutinine que le lait de vache ordinaire.

11. - Les sels du lait agissent pour retarder fortement l'agglutination des globules gras, et si la montée de la crème a lieu plus facilement dans le lait cru, quand on l'étend, la toute première cause de ce phénomène est qu'on diminue ainsi sa concentration saline.

12. - La viscosité du lait est par contre d'importance secondaire pour la montée de la crème, d'où il s'ensuit que le sérum ne laisse pas monter sa crème plus facilement que le lait et que, dans le mélange crème-eau, la crème ne se sépare pas du tout si on n'ajoute pas d'agglutinine, ainsi que son complément. 
13. - Si dans le lait ayant subi Ia pasteurisation basse, l'addition d'eau agit pour retarder la montée de la crème, e'est parce que l'ogglutinine est déjà, dans un tel lait, tellement détruite, qu'une nouvelle diminution de sa quantité n'est pas supportable.

14. - Dans le lait et dans le sérum qui contiennent de l'albumine coagulée, la présence de ce produit agit au plus haut degré pour faciliter la séparation de la crème. En ce qui concerne le lait, ceci n'est, du reste, exact que quand il est additionné d'eau.

15. - Un chauffage de 5 minutes à $68^{\circ} \mathrm{C}$. détruit à peu près autant de bactéries qu'une demi-heure de chauffage à $63^{\circ} \mathrm{C}$. Les bactéries de la tuberculose sont rendus inoffensives par les 2 méthodes de pasteurisation.

16. - Le nombre de bactéries qui survivent à la pasteurisation basse du lait dépend surtout de l'espèce à laquelle elles appartiennent, et est presque incépendant de la teneur en bactéries originale du lait. Le pour mille des survivants qui, pour la pasteurisation lente, varie entre 0,1 et 30 est, par conséquent, une mauvaise représentation de l'effectivité de la pasteurisation.

17. - La pasteurisation basse détruit pratiquement toutes les bactéries (à l'exception de certains microcoques) qui peuvent se développer à. basse température, et le lait ayant été soumis à la pasteurisation basse se conserve par conséquent presque aussi bien que le lait ayant été soumis à la pasteurisation haute, si seulement on le maintient à basse température ( $5^{\circ} \mathrm{C}$. ou en-dessous).

18. - Les pouvoirs bactéricides du lait sont bien plus aptes à résister qu'on ne le croit généralement. Ils ne sont ébrantés que par la pasteurisation haute et sont spécialement actifs dans le lait ayant subi la pasteurisation basse, de sorte que celui-ci conservé à $5^{\circ} \mathrm{C}$. peut arriver à contenir beaucoup moins de bactéries que tout de suite après la pasteurisation. Comme dans les villes, il se passe toujours quelque temps avant que le lait pasteurisé ne soit vendu, cette eirconstance n'est pas sans importance pratique.

19. - Les pouvoirs bactéricides du lait semblent se composer de plusieurs composants cifférents, et nous nous réservons de revenir sur cette question dans une étude spéciale.

\section{(Traduction C. Wolf.)}

(Fin)

Erratum : p. 623 , lire avortement épizootique au lieu de jetage des veaux infectieux. 